\section{Nachruf \\ Prof. Dr. Walter H. Hörl 11.7.1945 - 25.6.2013}

Prof. Dr. Walter H. Hörl, der Ordinarius für Nephrologie der Medizinischen Universität Wien und Leiter der Abteilung für Nephrologie und Hämodialyse des Allgemeinen Krankenhauses der Stadt Wien ist am 25. Juni 2013 kurz vor seinem 68. Geburtstag und kurz vor seiner Emeritierung verstorben.

Prof. Hörl stammt aus dem Allgäu und promovierte in Würzburg zum Doktor der Medizin. Anschließend erwarb er in Bochum ein Doktorat in Biochemie. In Würzburg hatte er dann die Ausbildung zum Internisten und Nephrologen abgeschlossen. Von dort ist Hörl nach Freiburg gegangen, wo er als leitender Oberarzt die Nephrologie betreut hat. 1990 wurde er zum Ordinarius für Nephrologie an die Universität Homburg/Saar berufen.

Prof. Hörl ist 1992 zum ersten österreichischen Ordinarius für Nephrologie an der Medizinischen Fakultät - später Medizinische Universität Wien berufen worden und hat die Leitung der Abteilung für Nephrologie am Allgemeinen Krankenhaus der Stadt Wien übernommen und seither ausgeübt.

In dieser Zeit hat Hörl grundlegend die Österreichische Nephrologie gestaltet, die Nephrologie am Allgemeinen Krankenhaus aufgebaut und das Fach „Nephrologie“ in seiner Breite zu dem gemacht, in der es sich heute präsentiert. Die wichtigsten nephrologischen Positionen in ganz Österreich wurden mit Personen aus seinem Mitarbeiterkreis besetzt.

Prof. Hörl war ein bis zuletzt aktiver, erfolgreicher und international hoch geehrter Wissenschafter und medizinischer Forscher. Er hat mehr als 600 Originalarbeiten und unzählige Buchbeiträge verfasst, mehrere Bücher herausgegeben und ist einer der international gefragtesten nephrologischen Redner gewesen. Er war Gutachter und im Editorial Board vieler internationaler Fachzeit- schriften und war auch selbst als Herausgeber von Zeitschriften erfolgreich.

Seit 1998 war Hörl Member of the Royal College of Physicians (FRCP). Hörl war Präsident und auch Ehrenmitglied verschiedener nationaler und internationaler Fachgesellschaften. Für seine beeindruckenden Leistungen wurde Prof. Hörl mit mehreren hochrangigen internationalen Preisen ausgezeichnet $u$. a. mit der Franz-Volhard-Medaille, mit dem Nils-Alwall-Preis, dem Addis Award, der International Distinguished Research Medal (National Kidney Foundation, USA) und der Albert Struyvenberg Medal (European Society of Clinical Investigation).

Prof. Hörl war nicht nur ein herausragender Wissenschafter, sondern auch ein begeisteter und begeistender, von seinen Studenten verehrter Lehrer. Er war ein fürsorglicher, empathischer Arzt, der für seine Patienten da war, ihnen mit unendlicher Geduld zugehört hat. Prof. Hörl war ein verständnisvoller, humorvoller und toleranter Chef, der für seine Mitarbeiter immer ansprechbar war. Auch oder gerade als Mensch und Arzt war er für uns alle ein Vorbild, ein „Mann für jede Jahreszeit“ mit einem beeindruckenden Berufsethos.

Die Medizinische Universität Wien und das Allgemeine Krankenhaus der Stadt Wien verlieren mit Prof. Hörl einen ihrer verdienstvollsten, profiliertesten und einflussreichsten Mitarbeiter. Unser Mitgefühl ist bei seiner Frau und seinen drei Söhnen. Wir werden Prof. Hörl nicht nur in dankbarer und ehrender Erinnerung haben sondern uns bemühen sein Vermächtnis, sein Werk weiterzuführen und weiter zu entwickeln.

\section{Prof. Dr. Wilfred Druml}

Für die Mitarbeiter der Abteilung für Nephrologie und Hämodialyse

Allgemeines Krankenhaus der Stadt Wien 